\title{
Development and internal validation of a predictive risk model for anxiety after completion of treatment for early stage breast cancer
}

\author{
Jenny Harris ${ }^{1 *}$ (D, Edward Purssell ${ }^{2}$, Victoria Cornelius ${ }^{3}$, Emma Ream $^{1}$, Anne Jones $^{4}$ and Jo Armes ${ }^{1}$
}

\begin{abstract}
Objective: To develop a predictive risk model (PRM) for patient-reported anxiety after treatment completion for early stage breast cancer suitable for use in practice and underpinned by advances in data science and risk prediction.

Methods: Secondary analysis of a prospective survey of $>800$ women at the end of treatment and again 6 months later using patient reported outcome (PRO) the hospital anxiety and depression scale-anxiety (HADS-A) and $>20$ candidate predictors. Multiple imputation using chained equations (for missing data) and least absolute shrinkage and selection operator (LASSO) were used to select predictors. Final multivariable linear model performance was assessed $\left(R^{2}\right)$ and bootstrapped for internal validation.

Results: Five predictors of anxiety selected by LASSO were HADS-A (Beta $0.73 ; 95 \% \mathrm{Cl} 0.681,0.785$ ); HAD-depression (Beta 0.095; 95\% Cl 0.020, 0.182) and having caring responsibilities (Beta 0.488; 95\% Cl 0.084, 0.866) increased risk, whereas being older (Beta $-0.010 ; 95 \% \mathrm{Cl}-0.028,0.004$ ) and owning a home (Beta $0.432 ; 95 \% \mathrm{Cl}-0.954,0.078$ ) reduced the risk. The final model explained $60 \%$ of variance and bias was low ( -0.006 to 0.002$)$.

Conclusions: Different modelling approaches are needed to predict rather than explain patient reported outcomes. We developed a parsimonious and pragmatic PRM. External validation is required prior to translation to digital tool and evaluation of clinical implementation. The routine use of PROs and data driven PRM in practice provides a new opportunity to target supportive care and specialist interventions for cancer patients.
\end{abstract}

Keywords: Anxiety, Patient reported outcomes, Breast cancer, Predictive risk models, Cancer survivors, Supportive care

\section{Introduction}

Symptoms of anxiety are frequently reported in response to breast cancer diagnosis and treatment [1]. Around 18$33 \%$ of women will experience anxiety following diagnosis of cancer [2,3] and this may persist in $25 \%$ of women living with and beyond breast cancer (hereafter referred to as breast cancer survivors, BCS) two to four years after diagnosis, and in $15 \%$ after five [4]. Whilst internationally

\footnotetext{
* Correspondence: jen.harris@surrey.ac.uk

'School of Health Sciences, Faculty of Health and Medical Sciences, University of Surrey, Kate Granger Building, Priestley Road, Guildford, Surrey GU2 7YH, UK

Full list of author information is available at the end of the article
}

the importance of risk assessment for follow-up care is increasingly recognized $[5,6]$, there has been few attempts to identify those likely to experience late emotional and psychological effects amongst the growing number of cancer survivors and little application of modern approaches to data science and predictive risk modelling $[7,8]$. In many countries follow-up care has shifted away from specialist cancer settings towards self-care and communitybased services for BCS at low risk of cancer recurrence and late effects (physical and psychological) [7]. Given these changes, one approach to ensure psychosocial distress is assessed and addressed would be to use patient reported outcomes (PRO) alongside routinely collected data 
during cancer treatment to help identify those at increased risk of anxiety, and related conditions, long-term to further tailor supportive care services.

Predictive risk models (PRMs) aim to predict the risk of having or developing an outcome based on multiple variables [9]. To date studies using best practice approaches to PRM development have primarily focused on developing models for clinical outcomes [10]. PRMs have been developed and applied to inform screening, care and treatment recommendation in areas as diverse as organ transplantation [11], cancer [12, 13] and cardiovascular disease [14]. Development of a PRM of anxiety in BCS could be used to enhance follow-up care through heightened clinical awareness [10] [7]. However, although cancer policy identifies the need to offer extra support for high risk conditions such as anxiety $[15,16]$ as yet validated PRM stratification tools are lacking [7] and so are not routinely used.

Factors predictive of anxiety after completion of breast cancer treatment remain under-researched, use crosssectional data [8] and are inadequately assessed [15], with little application of best practice guidance in the modelling process [17] [9, 18]. Traditional approaches to regression modelling focus on explaining phenomena rather than the ability to generate robust predictions $[9,18,19]$. This is common practice in psychosocial research where models are typically developed based on stepwise methods or univariate screening (typically including a variable in a model if it reaches a certain threshold of probability) [19]. Whilst such approaches attempt to reduce bias to obtain representations of the underlying data, this can lead to overfitting of the model to the data and reduce predictive performance in new data $[20,21]$. If the goal is developing predictive risk models, statistical methods are required that minimize the combination of bias and estimation variance, occasionally sacrificing accuracy for improved precision and utility $[9,19]$. A robust and useable PRM is generalizable, not overfitted to the data, and limits the number of variables that are required to be collected in routine practice $[9,19]$. Applying robust methods to develop data driven and powerful PRM for psycho-oncology is paramount if we are to target on-going assessment and support in survivorship to those at greatest risk.

This study aimed to develop a predictive risk model, using principles of statistical learning to ensure robustness, [17] to determine the probability of experiencing anxiety in women with early stage breast cancer, to estimate the model's predictive performance and undertake internal validation.

\section{Methods}

\section{Study design}

Secondary data analysis of a longitudinal cohort, the Supportive Care Needs Study (SCNS) of people receiving treatment with curative intent. This secondary analysis focused on women diagnosed with breast cancer only [22]. Ethical approvals were obtained as part of the original study.

\section{Participants and procedures}

Sixty-six cancer centres in England participated in the SCNS during 2005. Eligible patients were: $\geq 18$ years; able to read English; undergoing curative treatment; women diagnosed with breast cancer. Nurses recruited consecutive eligible patients. Participants self-completed a postal survey after their final treatment (T0), and 6 months later (T1), with non-response reminders. Previously published work suggests the sample was representative of cancer centres in the UK [22].

\section{Outcome measure}

The PRO was Hospital Anxiety and Depression Scale (HADS)-Anxiety (HADS-A) [23] total sub-scale score 6months after treatment completion (T1). HADS-A includes seven items to compute a total anxiety score (range 0-21). In clinical practice HADS-A is widely used as a screening tool and is both valid and reliable for identifying symptoms of anxiety in cancer and other clinical populations [24, 25].

\section{Potential predictors}

Selection of predictors for inclusion in the model was informed by results from a systematic review [8], written feedback from people with cancer contacted through a patient advocacy group and an expert advisory group. They provided feedback on potential ease of use in clinical settings (e.g. are data routinely collected or easily accessible?) and patient views (e.g. is it acceptable?), an important consideration if a PRM is to be implemented in routine clinical practice [10]. An overview of candidate predictors considered in the model is provided in Table 1. These included socio-demographic (age, marital status, caring responsibilities, employment status, highest educational level, car ownership, housing tenure, self-reported financial strain), psychological (prior poor mental health as indicated by T0 HADS) and clinical variables (comorbidities, disability, type of cancer treatment, self-report of feeling sick, fatigue or pain). Several pre-determined interactions based on previous evidence were considered including symptoms (pain, fatigue and depression) [26] and economic factors (economic status, homeownership and financial strain) [27].

\section{Statistical analysis}

Continuous predictors including age, HADS-A and HADS-Depression (HADS-D) were used to retain maximum predictive information [28] and for later translation of the tool into different clinical practices where appropriate risk grouping can be determined [29]. No 
Table 1 Sample characteristics

\begin{tabular}{ll}
\hline Candidate predictors & N (\%) \\
\hline Socio-demographic
\end{tabular}

Age:

19-51 years
52-59 years
60-65 years
66-71 years
72+ years
Mean age
Missing

$250(31.1)$
$192(23.9)$
$150(19.7)$
$109(13.7)$
$102(12.7)$
$58.0($ SD 11.5)
(range 27-88)
$13(1.6)$

Marital status

Married or living with partner

590 (72.5)

Widowed

$92(11.3)$

Divorced / Separated

Single

Missing

White British ethnicity

Missing

Lives alone

Missing

Housing tenure

Owner-occupier

Renting

Other

Missing

Have any caring responsibilities

Missing

84 (10.3)

$48(5.9)$

$2(2.3)$

761 (93.6)

3 (0.4)

$140(17.4)$

$11(1.4)$

694 (85.3)

$104(12.8)$

$16(2.0)$

$2(0.3)$

$227(28.5)$

$19(2.3)$

Highest level of qualification

No formal qualification

286 (35.6)

A level or equivalent

GCSE/O Level

97 (12.1)

$242(30.1)$

Degree/higher degree

Missing

$178(5.1)$

$13(1.6)$

\section{Employment status}

Working

268 (33.2)

On leave

$130(16.1)$

Retired

$329(40.7)$

Not working

$81(9.5)$

$8(0.3)$

$709(87.0)$

Use of car or van

Missing

Clinical

Mean HADS-A (baseline)

6.5 (SD 4.2,

14 (1.4)
Table 1 Sample characteristics (Continued)

\begin{tabular}{|c|c|}
\hline Candidate predictors & $\mathrm{N}(\%)^{\mathrm{a}}$ \\
\hline Mean HADS-D (baseline) & $\begin{array}{l}3.5 \text { (SD 3.2, } \\
\text { median 3.0, IQR 4.0) }\end{array}$ \\
\hline Missing & $4(0.8)$ \\
\hline Moderate or severe need: feeling unwell & $64(8)$ \\
\hline Missing & $15(1.8)$ \\
\hline $\begin{array}{l}\text { Moderate or severe need: lack of } \\
\text { energy/fatigue }\end{array}$ & $164(20.3)$ \\
\hline Missing & $8(1.0)$ \\
\hline Moderate or severe need: feeling pain & $70(8.7)$ \\
\hline Missing & $14(1.7)$ \\
\hline Longstanding comorbid illness & $317(39.2)$ \\
\hline Missing & $7(0.9)$ \\
\hline \multicolumn{2}{|l|}{ Cancer-related treatments } \\
\hline Chemotherapy & $350(64.6)$ \\
\hline Missing & $274(33.6)$ \\
\hline Radiotherapy & $766(96.6)$ \\
\hline Missing & $23(2.8)$ \\
\hline Hormone therapy & $537(69.3)$ \\
\hline Missing & $41(5.0)$ \\
\hline \multicolumn{2}{|l|}{ Outcome } \\
\hline Mean HADS-A ${ }^{b}$ & $6.8(S D$ 4.4) \\
\hline Missing & $150(18.4)$ \\
\hline
\end{tabular}

${ }^{a}$ Candidate predictor counts and percentages are for valid responses, except for missing data which represents overall figure

${ }^{\mathrm{b}}$ Values for MI data. Complete case values were mean 6.7 (SD 4.3, median 6.0, IQR 7.0)

author guidance exists for handling missing values in the HADS questionnaire, so a pragmatic approach was adopted in line with recent evidence [30]. If an item was missing for HADS sub-scale all other scores for that participant were used to impute the mean value for the missing item [30]. If $>2$ items were missing the whole HADS sub-scale was treated as missing. We examined associations with missingness using a series of logistic regressions to inform selection of variables included in the imputation models [31].

Missing data were handled using multiple imputation using chained equations (MICE) [32] to impute 50 datasets $[32,33]$ with predictive mean matching for continuous data (supplement 1). HADS scores had an approximately Gaussian distribution and extensive assessment was undertaken of model specifications for both the imputation and main analysis models. For example, collinearity was explored using variance inflation factors, margins, margins plots and contrasts (data available upon request). median 6.0, IQR 6.0)

\section{Selection of candidate predictors}

Stakeholder involvement highlighted that the potential list of $>20$ predictors would be unwieldly to use in 
clinical practice and unacceptable to patients, so this needed to be reduced. Traditionally analysts used univariate screening or stepwise regressions to achieve this, where inclusion of variables in a model is overdependent on null hypothesis significance testing and based on predetermined criteria (e.g. $p$ values) [19]. However, it is now widely accepted that this should be avoided because it can lead to poor estimation, is biased in selection and can result in model overfitting [19, 34]. These weaknesses limit the generalizability of the resulting regression model when intended for prediction purposes [21,35].

Modern regularization techniques provide a powerful alternative to overcome these problems [21]. In this secondary analysis Least Absolute Shrinkage and Selection Operator (LASSO) [36] was used as it produces parsimonious models and can help to minimize prediction error and overfitting by reducing the regression coefficients. This is achieved by introducing a penalty term $[19,21,37]$ whereby the penalty term is equal to the sum of the absolute coefficient, meaning all coefficients are shrunk and some reduce to zero [38]. Those reduced to zero are effectively removed from the model, making it a useful tool for developing parsimonious PRM to be used in clinical practice. To put simply, LASSO introduces some constraint which prevents the coefficients estimates having an inappropriately large magnitude [39].

LASSO was implemented for each imputed dataset, with predictors selected if they were included in $\geq 50 \%$ of the models $[32,40]$ or if they were a known predictor (age, anxiety and depression) [8]. LASSO was performed for all candidate predictors and again separately for all candidate predictors plus interactions. The final model's predictive performance was internally validated using Bootstrapping based on MI dataset. By generating a new sample of data from the original sample this provides estimates to account for model overfitting or uncertainty in the entire model development process [17]. Bootstrap distribution for the predictors across 1000 results was compared to those of the original model. Model discrimination was assessed by the explained variance $\left(R^{2}\right)[19]$. All analyses followed recommendations for multivariable predictive research $[17,35,41,42]$. To compare with standard approaches to model development we also built a model using univariate screening on the complete-case data, whereby a candidate predictor was included in the multivariable model if unadjusted $p \leq 0.10$ [19]. Data analysis was performed using Stata (version 15) [43].

\section{Results}

There were 1847 people who agreed to participate in the original study and 1425 returned the initial survey (79\%). The sample included 816 women with breast cancer who were included in this study, of whom 674 returned surveys at T1 (Fig. 1).
Participant characteristics are presented in Table 1. Mean age was 58 years (SD 11.51), most were married or living with a partner (72.5\%) and White British (93.6\%). Mean anxiety score was 6.5 (SD 4.2) at T0 and 6.8 (SD 4.34) at $\mathrm{T} 1$.

\section{Predictive risk model of anxiety}

From the 20 candidate predictors, five were selected by LASSO in $>50 \%$ of the MI datasets (Fig. 2, supplement 2) [40]. When examining the LASSO including additional interactions, two additional predictors were selected (financial strain and working status; working status and homeownership), however, inclusion of these did not improve model performance and so the parsimonious five predictor model was chosen (not presented but available on request).

The final model predicted that $\mathrm{T} 1$ anxiety scores are higher with increasing scores on T0 HADS-A, HADS-D and having caring responsibilities but decrease with older age and being a homeowner (Table 2). The final model explained $60 \%$ variance in the data. Bootstrap validation results suggest the level of bias for the final model was low ( -0.006 to $0.002 \%$, Table 2$)$, indicating stable accuracy of the estimate between the original and bootstrap samples. In comparison the univariate screening approach resulted in the inclusion of ten predictors (age, living alone, caring responsibilities, homeowner, financial need, employment status, lack of energy/fatigue, feeling unwell, HADS-A, HADS-D) and explained 59\% of variance in the data (supplement 3).

\section{Discussion}

This study developed a parsimonious PRM for anxiety 6-months following breast cancer treatment completion, using LASSO regression to identify the most salient predictors. Few studies have attempted to develop multivariable predictive risk models for patient reported anxiety breast cancer treatment [8]. Anxiety at the end of treatment was an important predictor of anxiety 6-months later, a finding that is supported by previous research [8] and policy [16]. In this study we used HADS-A, but other screening tools can be used in practice with similar performance [35, 36]. Good screening tools are typically quick to administer, for example HADS takes $2-5$ min to complete [37], and advances in digital data collection mean this can be linked with other important predictive data in real-time.

In psycho-oncology existing models of outcomes tend to be explanatory, attempting to elucidate causal mechanisms. Here the goal was prediction and so we used a data driven approach. We intended the model to be practical; for it to be useful in clinical practice it needed to include variables that are either routinely collected or 


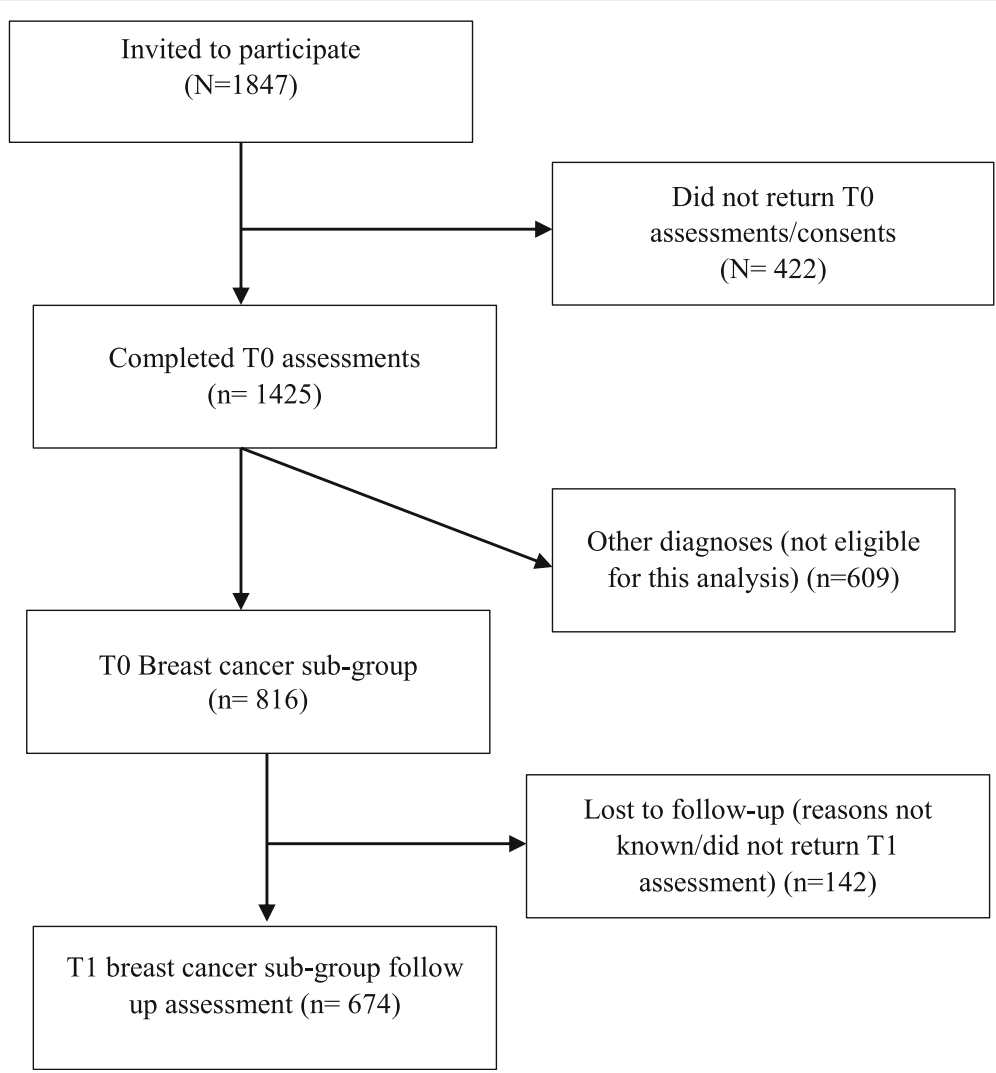

Fig. 1 Flow chart of participants' study inclusion. T0, baseline (at the end of treatment); T1, 6 months after baseline

required limited additional information to be collected. In contrast, the model built using traditional univariate screening resulted in twice the number of predictors being including in the model and would require greater clinical effort and resource to collect.
We found LASSO regression, increasingly used in machine learning and precision medicine but rare in psychooncology, was a helpful method to identify predictors and multiple imputation enabled us to fully utilize the dataset. We would encourage wider use of robust statistical

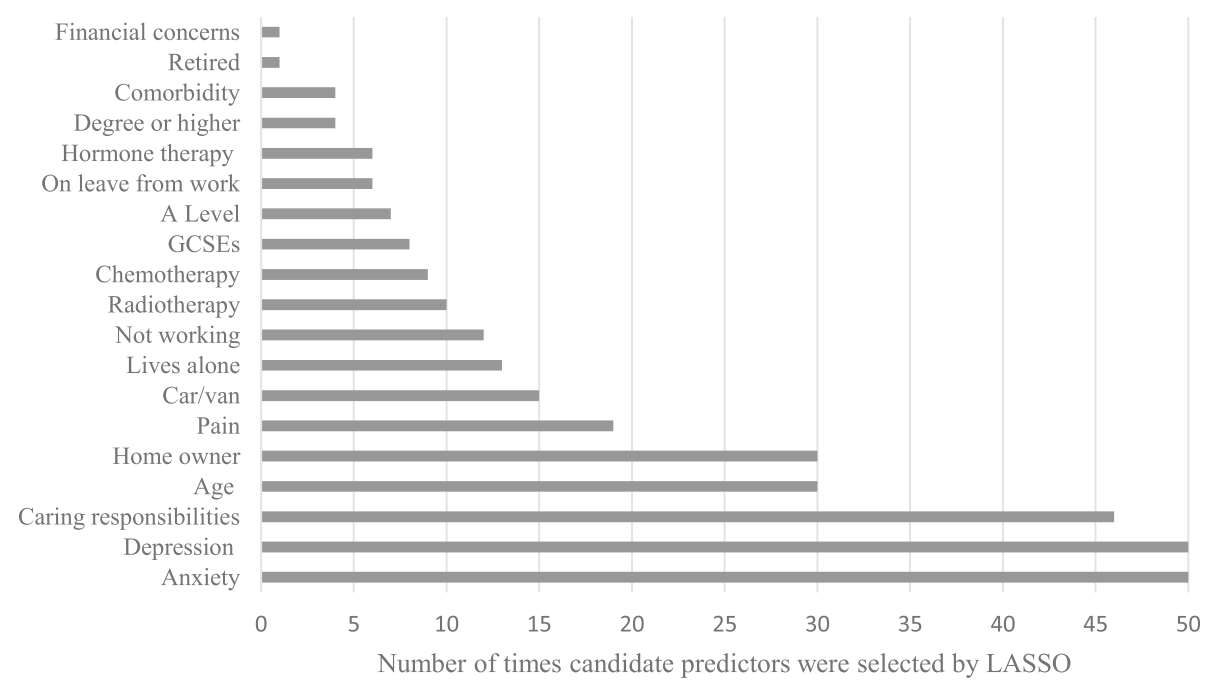

Fig. 2 Number of times candidate predictors were selected by LASSO in MI datasets $(m=50)$ 
Table 2 Prediction model estimates and bootstrap estimates

\begin{tabular}{|c|c|c|c|c|c|c|c|c|}
\hline \multirow[t]{2}{*}{ Predictor } & \multicolumn{3}{|c|}{ MI estimate } & \multicolumn{5}{|c|}{ MI bootstrap estimate } \\
\hline & B & SE & $95 \% \mathrm{Cl}$ & $B_{b}$ & $\mathrm{SE}_{\mathrm{b}}$ & $\mathrm{Z}$ & Bias & B 95\% Cl \\
\hline HADS-A & 0.734 & 0.034 & $0.67,0.80$ & 0.734 & 0.027 & $27.12^{a}$ & 0.000 & $0.68,0.79$ \\
\hline HADS-D & 0.094 & 0.043 & $0.01,0.18$ & 0.095 & 0.041 & $2.28^{\mathrm{b}}$ & 0.001 & $0.02,0.18$ \\
\hline Age & -0.011 & 0.011 & $-0.03,0.01$ & -0.010 & 0.008 & -1.30 & 0.001 & $-0.03,0.01$ \\
\hline Caring responsibility & 0.485 & 0.274 & $-0.05,1.03$ & 0.488 & 0.200 & $2.43^{\mathrm{a}}$ & 0.002 & $0.08,0.87$ \\
\hline Homeowner & -0.426 & 0.326 & $-1.07,0.22$ & -0.432 & 0.263 & -1.62 & -0.006 & $-0.95,0.08$ \\
\hline Constant & 2.515 & 0.793 & $0.95,4.08$ & 2.475 & 0.587 & $4.28^{\mathrm{b}}$ & -0.040 & $1.39,3.67$ \\
\hline
\end{tabular}

MI estimate: $\mathrm{B}=\mathrm{Ml}$ observed coefficient, $\mathrm{SE} \mathrm{B}=$ standard error of $\mathrm{B}, 95 \% \mathrm{Cl}$ (confidence intervals)

MI Bootstrap estimate: $B_{b}=$ MI bootstrap estimates of coefficient, $S_{b}=$ standard error of $B_{b}, z=$ bootstrap estimate divided by the standard error, bias = bias for the parameter estimate, $\mathrm{B} 95 \% \mathrm{Cl}=$ bias corrected $95 \% \mathrm{Cl}$

All estimates are based on $\mathrm{Ml}$ data $(\mathrm{M}=50)$ and Bootstrap distribution across 1000 results $\left(10,000\right.$ random samples with replacement) ${ }^{\mathrm{a}} p<0.05{ }^{\mathrm{b}} p<=0.001$ Simple 6-month predicted anxiety $=2.5+($ HADS-A score $\times 0.7)+($ HADS-D score $\times 0.1)+($ age $x-0.1)+0.4$ (if carer) +-0.4 (if homeowner)

techniques like these for data driven model development for psychological outcomes in medicine. LASSO allowed us to identify the most relevant predictors of anxiety at follow-up and develop a parsimonious PRM.

An important aspect of predictive rather than explanatory modelling is that variables included in risk models will not necessarily be individually statistically significant [21]. However, they may still be important to include to fine-tune performance as the aim is not hypothesis testing [19]. Thus, they can have clinical significance even in the absence of statistical significance. Younger age was identified as a possible predictor of anxiety after treatment finished in previous research [8]. Although only weakly associated here, age was still selected by the LASSO procedure. Social risk factors, although generally thought to be predictive of anxiety for people with other chronic health conditions [44], have not been consistently identified in BCS [8]; using LASSO the small but important effects of social factors helped us refine the model. Housing tenure and having caring responsibilities have not been identified as predictors in previous research and it may be that specific predictors are more relevant to certain social, economic and cultural contexts. For example, in the UK homeownership may serve as a proxy for social economic status but may not be an important predictor in locations where renting is more widespread. Further, the definition of caring responsibility was quite broad as it could include any caring for an adult or child. Future research is needed to determine if it is caring per se, or whether it is responsibility for an adult, child or both that is important. There is some evidence that the risk of anxiety is greater during chemotherapy treatment than other treatment modalities [45]. However, in line with previous research [8], chemotherapy did not predict anxiety after treatment completion. This finding is an important message for patients and clinicians.

\section{Limitations}

This study was limited to secondary analysis of a preexisting data. Longitudinal research is expensive and funding scarce, so it is generally recognized that there is a need to use existing datasets for predictive modelling [46] and that data should ideally be observational and comprehensive, as with the current study. Nonetheless, there may be other important variables (lifestyle factors, newer treatments and adverse-events) not measured in primary research that may impact on anxiety. Indeed, these could be important candidate predictors to consider in future research to account for the variance unexplained in the model. However, this study completes the first stage in a development process that we will build upon in future model validation studies. Furthermore, a third of chemotherapy treatment data was missing which may present some reporting bias although we attempted to mitigate this using MICE. It was an advantage that this study used data that is widely collected and a tool that is well understood by clinicians and researchers.

The original research was conducted with a sample of women with early stage disease and good oncological prognosis. Results may be different for women with later stage disease, many of whom will live for many years with incurable disease. Further research is needed to determine predictors in this group. The study sample was limited to include only women who could read English and future research is needed to examine its predictive performance in across the diverse communities in the UK and its utility in international clinical contexts. Indeed, these issues have been noted as limitations of well-known PRM in cancer such as 'Adjuvant' and MammaPrint' [47].

To maintain predictive sensitivity the model was developed using a continuous outcome making immediate clinical interpretation less straightforward than if a model with a dichotomous outcome had been developed. 
However, this was deliberate, and we feel necessary, given the early stage of evidence in this area and to make the model more sensitive. Further, digital technologies allow greater ease of use and interpretation of such outcomes. Another limitation was the preliminary nature of this study and the PRM requires validation in an external sample with decision-curve analysis to determine the clinical impact of different thresholds [48]. Validation of this model in external samples will help us to identify individuals at low, moderate or high risk of anxiety after treatment completion, through calculation of risk scores/ algorithms, ready for translation into a digital tool and evaluation of clinical implementation in future research. High quality longitudinal data from similar cohorts is expensive to collect and currently scarce, therefore we plan to undertake secondary analysis of trials using PROs to further refine and externally validate the model.

Future research is needed to determine appropriate cut-offs based on best practice regarding predictive accuracy and clinical utility [21], and whether standard HADS-A thresholds are appropriate for this population [24] and adaptions using different outcome measures. For example, by examining the utility of different screening tools where it might be possible to calibrate and adapt PRM depending on the preferred instrument already used in clinical setting. Long-term such tools may help health services plan their resourcing and provision of follow-up care according to patient characteristics, permitting stratified follow-up with different support options for different levels of risk.

A strength of this study was the modelling strategy, it followed recommendations to limit bias in the identification of predictors. For example, in many studies model building procedures such as variable selection is undertaken on complete-case datasets, even where MI has been used. Current guidelines do not support this approach as the results can be biased and lack power [32, 40].

\section{Conclusions}

A myriad of individual predictors of anxiety for breast cancer survivors have been identified in previous research. It is impractical and unnecessary for these all to be collected and entered into a digital PRM in clinical practice by busy specialist cancer nurses or oncologists often responsible for referring to supportive or specialist psycho-oncology services. Further, previous research has not used sophisticated statistical learning approaches now recommended for developing PRM. This study developed a parsimonious PRM for anxiety after breast cancer treatment that, if further validated and refined, has the potential to be adapted to a digital tool to be used in clinical practice. At completion of treatment anxiety, depression and having caring responsibilities increased risk for anxiety, whereas being older and owning a home were protective. The methods presented here may provide a useful framework for others wanting to harness the power of data driven predictive models for psychological patient reported outcomes in clinical populations. PRMs present the opportunity to facilitate the use of routinely collected patient reported predictors and outcomes to enhance patient quality of life through stratified supportive care packages.

\section{Supplementary information}

The online version contains supplementary material available at https://doi. org/10.1186/s41687-020-00267-w.

Additional file 1: Supplement 1. Summary of final imputation model specification. Supplement 2. Illustration of the LASSO estimates.

Supplement 3. Estimates for model selection using univariate screening.

\section{Acknowledgements}

We would like to thank Independent Cancer Patients' Voice and KCL's Supportive Cancer Care group for their useful feedback.

\section{Authors' contributions}

$\mathrm{JH}$ analyzed the data. VC reviewed and validated the analysis. All authors contributed to the interpretation. JA was responsible for the source data (SCNS) collection and design. JH drafted the manuscript and all authors read and commented on drafts. All authors read and approved the final manuscript.

\section{Funding}

$J \mathrm{H}$ was supported by the National Institute for Health Research (NIHR) Collaboration for Leadership in Applied Health Research and Care South London at King's College Hospital NHS Foundation Trust. The views expressed are those of the authors and not necessarily those of the NHS, the NIHR or the Department of Health.

\section{Availability of data and materials}

The dataset analysed during the current study are not publicly available because this was a secondary analysis but are available via the corresponding author on reasonable request with permission from Dr. Jo Armes.

\section{Ethics approval and consent to participate}

Multicenter Research Ethics Committee approval was granted (05/MRE12/21), and research governance approval was gained from participating sites for original data collection. Informed consent was obtained from all individual participants included in the study.

\section{Consent for publication}

Not applicable.

\section{Competing interests \\ None.}

\section{Author details}

${ }^{1}$ School of Health Sciences, Faculty of Health and Medical Sciences, University of Surrey, Kate Granger Building, Priestley Road, Guildford, Surrey GU2 7YH, UK. ${ }^{2}$ School of Health Sciences, City, University of London, London, UK. ${ }^{3}$ Imperial Clinical Trials Unit (ICTU), School of Public Health, Faculty of Medicine, Imperial College London, London, UK. ${ }^{4}$ Florence Nightingale Faculty of Nursing, Midwifery and Palliative Care, King's College London, London, UK. 
Received: 17 July 2020 Accepted: 8 November 2020 Published online: 04 December 2020

\section{References}

1. Schwarz, R., Krauss, O., Höckel, M., Meyer, A., Zenger, M., \& Hinz, A. (2008). The course of anxiety and depression in patients with breast cancer and gynaecological cancer. Breast Care, 3(6), 417-422 6

2. Zabora, J., BrintzenhofeSzoc, K., Curbow, B., Hooker, C., \& Piantadosi, S. (2001). The prevalence of psychological distress by cancer site. PsychoOncology., 10(1), 19-28.

3. Brintzenhofe-Szoc, K. M., Levin, T. T., Li, Y., Kissane, D. W., \& Zabora, J. R. (2009). Mixed anxiety/depression symptoms in a large cancer cohort: prevalence by cancer type. Psychosomatics, 50(4), 383-391. https://doi.org/ 10.1176/appi.psy.50.4.383

4. Burgess, C., Cornelius, V., Love, S., Graham, J., Richards, M., \& Ramirez, A. (2005). Depression and anxiety in women with early breast cancer: Five year observational cohort study. British Medical Journal, 330(7493), 702-705. https://doi.org/10.1136/bmj.38343.670868.D3

5. NCSI (2013). Living with and beyond cancer: Taking action to improve outcomes. London: NHS Improvement, Department of Health, Macmillan Cancer Support.

6. Richards, M., Corner, J., \& Maher, J. (2011). The National Cancer Survivorship Initiative: New and emerging evidence on the ongoing needs of cancer survivors. British Journal of Cancer, 105, S1-S4.

7. Watson, E., Rose, P. W., Neal, R. D., Hulbert-Williams, N., Donnelly, P. K., Hubbard, G., et al. (2012). Personalised cancer follow-up: Risk stratification, needs assessment or both? British Journal of Cancer, 106(1), 1-5.

8. Harris, J., Cornelius, V., Ream, E., Cheevers, K., \& Armes, J. (2017). Anxiety after completion of treatment for early-stage breast cancer: A systematic review to identify candidate predictors and evaluate multivariable model development. Supportive Care in Cancer., 25(7), 2321-2333.

9. Moons, K. G., Royston, P., Vergouwe, Y., Grobbee, D. E., \& Altman, D. G. (2009). Prognosis and prognostic research: what, why, and how? Bmi, 338, b375. https://doi.org/10.1136/bmj.b375.

10. Moons, K. G., Altman, D. G., Vergouwe, Y., \& Royston, P. (2009). Prognosis and prognostic research: Application and impact of prognostic models in clinical practice. Bmi., 338, b606.

11. Kim, W. R., Wiesner, R. H., Therneau, T. M., Poterucha, J. J., Porayko, M. K., Evans, R. W., et al. (1998). Optimal timing of liver transplantation for primary biliary cirrhosis. Hepatology., 28(1), 33-38.

12. Lee, A., Mavaddat, N., Wilcox, A. N., Cunningham, A. P., Carver, T., Hartley, S., et al. (2019). BOADICEA: A comprehensive breast cancer risk prediction model incorporating genetic and nongenetic risk factors. Genetics in Medicine, 21(8), 1708-1718.

13. Olivotto, I. A., Bajdik, C. D., Ravdin, P. M., Speers, C. H., Coldman, A. J., Norris, B. D., et al. (2005). Population-based validation of the prognostic model ADJUVANT! For early breast cancer. Journal of Clinical Oncology, 23(12), 2716-2725.

14. Ramsay, G., Podogrodzka, M., McClure, C., \& Fox, K. A. A. (2007). Risk prediction in patients presenting with suspected cardiac pain: The GRACE and TIMI risk scores versus clinical evaluation. QJM, 100(1), 11-18. https://doi. org/10.1093/qjmed/hcl133.

15. Milligan, F., Martinez, F., Aal, S. H. M. A., Ahmed, S. A., Joby, B., Matalam, J. S., et al. (2018). Assessing anxiety and depression in cancer patients. The British Journal of Nursing, 27(10), S18-S23.

16. Andersen, B. L., Rowland, J. H., \& Somerfield, M. R. (2014). Screening, assessment, and care of anxiety and depressive symptoms in adults with cancer: An American Society of Clinical Oncology guideline adaptation. Journal of Oncology Practice, 11(2), 133-134

17. Moons, K. G., Altman, D. G., Reitsma, J. B., loannidis, J. P., Macaskill, P Steyerberg, E. W., et al. (2015). Transparent reporting of a multivariable prediction model for individual prognosis or diagnosis (TRIPOD): Explanation and elaboration. Annals of Internal Medicine, 162(1), W1-W73.

18. Shmueli, G. (2010). To explain or to predict? Statistical Science, 25(3) 289-310.

19. Steyerberg, E. W. (2008). Clinical prediction models: A practical approach to development, validation, and updating. New York: Springer-Verlag.

20. Flom, P. L., \& Cassell, D. L. (2007). Stopping stepwise: Why stepwise and similar selection methods are bad, and what you should use. In NorthEast SAS Users Group Inc 20th Annual Conference: 11-14th November 2007.
Baltimore: Pacific Northwest SAS Users Group (PNWSUG). https://www lexjansen.com/pnwsug/2008/DavidCassell-StoppingStepwise.pdf

21. Harrell, F. (2015). Regression modeling strategies: With applications to linear models, logistic and ordinal regression, and survival analysis. Springer International Publishing. Springer Nature Switzerland AG.

22. Armes, J., Crowe, M., Colbourne, L., Morgan, H., Murrells, T., Oakley, C., et al. (2009). Patients' supportive care needs beyond the end of cancer treatment: a prospective, longitudinal survey. Journal of Clinical Oncology, 27(36), 61726179 8p. https://doi.org/10.1200/JCO.2009.22.5151.

23. Zigmond, A. S., \& Snaith, R. P. (1983). The hospital anxiety and depression scale. Acta Psychiatrica Scandinavica, 67(6), 361-370.

24. Singer, S., Kuhnt, S., Götze, H., Hauss, J., Hinz, A., Liebmann, A., et al. (2009). Hospital anxiety and depression scale cutoff scores for cancer patients in acute care. British Journal of Cancer, 100(6), 908-912.

25. Bjelland, I., Dahl, A. A., Haug, T. T., \& Neckelmann, D. (2002). The validity of the hospital anxiety and depression scale: An updated literature review. Journal of Psychosomatic Research, 52(2), 69-77.

26. Miaskowski, C., Cooper, B. A., Paul, S. M., Dodd, M., Lee, K., Aouizerat, B. E., et al. (2006). Subgroups of patients with cancer with different symptom experiences and quality-of-life outcomes: A cluster analysis. Oncology Nursing Forum, 33(5), e79-e89.

27. Saboonchi, F., Petersson, L.-M., Wennman-Larsen, A., Alexanderson, K., \& Vaez, M. (2015). Trajectories of anxiety among women with breast cancer: A proxy for adjustment from acute to transitional survivorship. Journal of Psychosocial Oncology, 33(6), 603-619.

28. Hendriksen, J., Geersing, G., Moons, K., \& De Groot, J. (2013). Diagnostic and prognostic prediction models. Journal of Thrombosis and Haemostasis, 11, 129-141.

29. Morse, R., Kendell, K., \& Barton, S. (2005). Screening for depression in people with cancer: The accuracy of the hospital anxiety and depression scale. Clinical Effectiveness in Nursing, 9(3), 188-196.

30. Bell, M. L., Fairclough, D. L., Fiero, M. H., \& Butow, P. N. (2016). Handling missing items in the hospital anxiety and depression scale (HADS): A simulation study. BMC Research Notes, 9(1), 479.

31. Manly, C. A., \& Wells, R. S. (2015). Reporting the use of multiple imputation for missing data in higher education research. Research in Higher Education, 56(4), 397-409.

32. White, I. R., Royston, P., \& Wood, A. M. (2011). Multiple imputation using chained equations: Issues and guidance for practice. Statistics in Medicine, 30(4), 377-399.

33. von Hippel, P. T. (2020). How many imputations do you need? A two-stage calculation using a quadratic rule. Sociological Methods \& Research, 49(3), 699-718. https://doi.org/10.1177/0049124117747303.

34. Whittingham, M. J., Stephens, P. A., Bradbury, R. B., \& Freckleton, R. P. (2006). Why do we still use stepwise modelling in ecology and behaviour? The Journal of Animal Ecology, 75(5), 1182-1189.

35. Hemingway, H., Croft, P., Perel, P., Hayden, J. A., Abrams, K., Timmis, A., et al. (2013). Prognosis research strategy (PROGRESS) 1: A framework for researching clinical outcomes. Bmj., 346, e5595.

36. Tibshirani R. Regression shrinkage and selection via the lasso. Journal of the Royal Statistical Society: Series B (Methodological). 1996:58(1):267-88. https://doi.org/10.1111/j.2517-6161.1996.tb02080.x

37. Steyerberg, E. W., Vickers, A. J., Cook, N. R., Gerds, T., Gonen, M., Obuchowski, N., et al. (2010). Assessing the performance of prediction models: a framework for some traditional and novel measures. Epidemiology (Cambridge, Mass.), 21(1), 128

38. Fonti, V., \& Belitser, E. (2017). Feature selection using LASSO. VU Amsterdam Research Paper in Business Analytics, 30, 1-25.

39. McNeish, D. M. (2015). Using lasso for predictor selection and to assuage overfitting: A method long overlooked in behavioral sciences. Multivariate Behavioral Research, 50(5), 471-484. https://doi.org/10.1080/00273171.2015. 1036965.

40. Wood, A. M., White, I. R., \& Royston, P. (2008). How should variable selection be performed with multiply imputed data? Statistics in Medicine, 27(17), 3227-3246.

41. Hingorani, A. D., van der Windt, D. A., Riley, R. D., Abrams, K., Moons, K. G. Steyerberg, E. W., et al. (2013). Prognosis research strategy (PROGRESS) 4: Stratified medicine research. Bmj., 346, e5793.

42. Steyerberg, E. W., Moons, K. G., van der Windt, D. A., Hayden, J. A., Perel, P., Schroter, S., et al. (2013). Prognosis research strategy (PROGRESS) 3: Prognostic model research. PLoS Medicine, 10(2), e1001381. 
43. Townsend, W. (2017). elasticregress. GitHub: Wilbur Townsend, Stanford University. https://github.com/wilburtownsend/elasticregress.

44. Remes, O., Brayne, C., Linde, R., \& Lafortune, L. (2016). A systematic review of reviews on the prevalence of anxiety disorders in adult populations. Brain and Behavior, 6(7), e00497.

45. Lim, C. C., Devi, M. K., \& Ang, E. (2011). Anxiety in women with breast cancer undergoing treatment: A systematic review. International Journal of Evidence-Based Healthcare, 9(3), 215-235.

46. Peat, G., Riley, R. D., Croft, P., Morley, K. I., Kyzas, P. A., Moons, K. G., et al. (2014). Improving the transparency of prognosis research: The role of reporting, data sharing, registration, and protocols. PLoS Medicine, 11(7), e1001671.

47. Engelhardt, E., Garvelink, M., Jd, H., van der Hoeven, J., Smets, E. M., Pieterse, A., et al. (2014). Predicting and communicating the risk of recurrence and death in women with early-stage breast cancer: A systematic review of risk prediction models. Journal of Clinical Oncology, 32(3), 238-250.

48. Vickers, A. J., \& Elkin, E. B. (2006). Decision curve analysis: A novel method for evaluating prediction models. Medical Decision Making, 26(6), 565-574.

\section{Publisher's Note}

Springer Nature remains neutral with regard to jurisdictional claims in published maps and institutional affiliations.

\section{Submit your manuscript to a SpringerOpen ${ }^{\circ}$ journal and benefit from:}

- Convenient online submission

- Rigorous peer review

- Open access: articles freely available online

- High visibility within the field

- Retaining the copyright to your article

Submit your next manuscript at $\boldsymbol{\nabla}$ springeropen.com 